\title{
Optimizing the Joint Replenishment and Channel Coordination Problem under Supply Chain Environment Using a Simple and Effective Differential Evolution Algorithm
}

\author{
Lin Wang, ${ }^{1}$ Hui Qu, ${ }^{1}$ Shan Liu, ${ }^{2}$ and Can Chen ${ }^{1}$ \\ ${ }^{1}$ School of Management, Huazhong University of Science \& Technology, Wuhan 430074, China
}

${ }^{2}$ Economics and Management School, Wuhan University, Wuhan 430072, China

Correspondence should be addressed to Shan Liu; 279266384@qq.com

Received 12 April 2014; Revised 11 May 2014; Accepted 17 May 2014; Published 17 June 2014

Academic Editor: Stefan Balint

Copyright (C) 2014 Lin Wang et al. This is an open access article distributed under the Creative Commons Attribution License, which permits unrestricted use, distribution, and reproduction in any medium, provided the original work is properly cited.

This paper presents a useful and practical procurement approach using the joint replenishment and channel coordination (JR-CC) policy in a two-echelon supply chain considering the coordination cost. The objective is to determine a basic replenishment cycle time and the replenishment interval to minimize the total cost of the supply chain. To solve this NP-hard problem, a simple and improved differential evolution algorithm (IDE) is developed. The performance of the IDE is verified by benchmark functions. Moreover, results of comparative numerical example show the effectiveness of the proposed IDE. IDE can be used as a good candidate for the JR-CC model. Results of numerical examples also indicate that the JR-CC policy can result in considerable cost saving, and enhance the efficiency of a supply chain. But not all members in the supply chain can benefit a lot using this policy. Moreover, results of sensitivity analysis show that retailers have more willingness to adopt the JR-CC policy than the manufacturers because of the different cost savings.

\section{Introduction}

Over the past few decades, the joint replenishment problem (JRP) has been heavily studied since not only is the JRP a multi-item inventory problem but also it is widely applied to lot sizing problems in manufacturing applications (Axsäter and Zhang [1]; Wang et al. [2]). The JRP means to group items into the same order from a supplier to achieve the purpose of sharing the main preparation costs and saving the procurement costs. Moreover, a quantity discount will be offered by the supplier when the order amount is greater than a predefined quantity. Olsen [3] developed an evolutionary algorithm to solve the JRP. Moon and Cha [4] studied the JRP with resource constraints. Khouja and Goyal [5] reviewed the literatures on JRPs from 1989 to 2005 and summarized heuristics for JRPs, special approaches to JRPs, and some special applications of JRPs. Since 2013, there are several relevant papers related to JRPs and extensions such as Qu et al. [6], Wang et al. [7, 8], Büyükkaramikli et al. [9], and Cui et al. [10]. However, these literatures mainly focus on the joint replenishment of multi-items from a single supplier in an individual enterprise.

In recent years, many companies realized that considerable cost savings can be achieved by the joint replenishment policy. Axsäter and Zhang [1] considered a two-level supply chain with a central warehouse and a number of identical retailers using the JR policy. Cha and Moon [11] and Moon et al. [12] developed efficient algorithms for solving the JRPs considering the quantity discounts. Cha and Park [13] dealt with the joint replenishment and delivery scheduling. Hsu [14] investigated the joint replenishment decisions involving combining different materials/components from several satellite factories to form a large shipment delivered to the central factory on a Just in Time (JIT) basis. T.-H. Chen 
and J.-M Chen [15] proposed four decision-making policies characterized by the joint replenishment and channel coordination (JR-CC) practice to determine optimal inventory replenishment and production policies in a supply chain. However, these literatures have not considered the potential coordination cost when the JR-CC policy is adopted in the supply chain. All the center of the supply chain in these literatures is the suppliers, because the suppliers can distribute the items to the retailers jointly to reduce the delivery cost. Moreover, the suppliers can provide quantity discounts to inspire the retailers to adopt the joint replenishment policy.

The aim of this paper is to study a useful and practical procurement approach using the JR-CC policy considering the coordination cost and provide a simple and effective solution. In this study, the advantage of joint replenishment and channel coordination (JR-CC) policy is investigated based on the work of Hsu [14]. Hsu [14] studied the joint replenishment decisions for a central factory and satellite factories under a Just in Time environment and regarded the members of the supply chain as the same benefit group. Adversely, we consider that they have independent financial accounting systems and make their own decisions from the view of retailers for the efficient implementation of JR-CC policy. Moreover, we further compare costs to obtain useful managerial insights. Because the success of the JR-CC policy needs a close cooperation with other enterprises, it can be regarded as a practical and effective management method for most enterprises who only concentrate on its own benefit without consideration for the others.

JRPs had been proven to be the NP-hard problems and they were rather hard to find effective algorithms (Cha and Moon [11]; Wang et al.[16, 17]). Hsu [14] designed a complex heuristic to handle this problem. Several heuristics may give acceptable solutions when the scale of optimization is small. However, it is not easy to find a proper heuristic with a robust performance. However, this heuristic is relatively complex for decision-makers and cannot handle JR-CC model with the cost constrain conveniently. With the increasing complexity of supply chain optimization problem (Geunes and Pardalos [18]; Geunes et al. [19]; Pardalos et al. [20]) and the development of the intelligent algorithms (Wang et al. [21]; Li et al. [22]; Wang et al., [23]; Cui et al. [24]), they were widely used for handling the practical supply chain optimization problems (Pei et al. [25]; Al-Anzi and Allahverdi [26]; Wang et al. [27]). Among these algorithms, the differential evolution algorithm (DE) had an extensive application in JRPs (Wang et al. $[2,6,8,9])$. So, a simple and effective DE is utilized to handle this problem. The DE was proposed by Storn and Price [28] for complex continuous nonlinear, nondifferentiable, and multimodal optimization problem. This technique combines simple arithmetic operators with the classical events of crossover, mutation, and selection to evolve from a randomly generated starting population to a final solution. Due to its simple structure, easy implementation, quick convergence, and robustness, the DE has been applied in a variety of fields (Al-Anzi and Allahverdi [26]; Pan et al. [29]; Qu et al. [6]). To the best of our knowledge, no work on the JR-CC model using DE can be found. The DE has a good performance in convergent speed, but the faster convergence may cause the diversity of population to descend quickly during the solution process. Moreover, a closely clustered population yields a premature convergence or a local optimum and cannot reproduce a better individual. So, the classic DE should be improved to find a good trade-off between convergence and diversity.

The rest of this paper is organized as follows. In Section 2, the independent replenishment (IR) model and JR-CC model are discussed. Section 3 proposes a DE-based solution for the proposed model. Section 4 contains numerical example and sensitivity analysis for the JR-CC models. Conclusions and future research are presented in Section 5.

\section{Mathematical Formulation}

2.1. Assumptions and Notations. We consider two-echelon supply chain consisting of a retailer replenishes $n$ materials from multiple manufacturers which distributes in a centralized location. The retailer and manufacturers share the information of demand and inventory, and both of them can hold inventory. We assume that the demand of retailer is constant and no shortage is allowed. The manufacturers are assumed to be a "make-to-order" producer, using a lot-for-lot policy to fulfill customer demand. Each time the retailer has a need for replenishment, a major ordering cost, regardless of the number of the materials included, and a minor ordering cost, related to material, are incurred. In each production run, the manufacturers have a constant production rate and spend corresponding setup cost. The quantity in one product cycle of material $i$ is split into multiple equal-size shipment lots delivered to the retailer.

The following notations are used:

$n$, number of materials involved in the model;

$i$, index of item, $i=1,2, \ldots n$;

$D_{i}$, demand rate of material $i$;

$P_{i}$, production rate of material $i$ in a manufacturer, $P_{i} \geq D_{i}$;

$k_{i}$, the number of shipments in which the material $i$ is delivered from the manufacturers to the retailer within one production cycle, a positive integer;

$S_{i}$, setup cost of material $i$ for the manufacturers;

$F$, major ordering cost for the retailer;

$f_{i}$, minor ordering cost of material $i$ for the retailer;

$H_{c i}$, carrying cost rate of material $i$ for the retailer;

$H_{s i}$, carrying cost rate of material $i$ for the manufacturers;

$T$, the common shipment cycle time for the $n$ materials;

$T_{i}$, the shipment cycle time of the material $i$ with independent replenishment;

$\mathrm{TC}_{s i 0}$, total relevant cost of material $i$ per year for the manufacturers with independent replenishment;

$\mathrm{TC}_{c 0}$, total relevant cost of $n$ materials per year for the retailer with independent replenishment; 
$\mathrm{TC}_{s i}$, total relevant cost of material $i$ per year for the manufacturers with joint replenishment and channel coordination;

$\mathrm{TC}_{c}$, total relevant cost of $n$ materials per year for the retailer with joint replenishment and channel coordination;

$\mathrm{JTC}_{0}$, total relevant cost for the supply chain per year with independent replenishment of materials;

JTC, total relevant cost for the supply chain per year with joint replenishment of materials and channel coordination.

Two models are discussed; one is independent replenishment (IR) model, in which the materials have their own shipment cycle time $T_{i}$; the other is JR-CC model, in which the materials have a common shipment cycle time $T$.

2.2. Formulation of the IR Model. With the independent replenishment policy, each entity in the supply chain concentrated on minimizing its own costs without considering the others. The retailer makes the replenishment decision for each item based on an EOQ policy (J.-M Chen and T.-H Chen [30]). Then the total cost of $n$ items per year for the retailer is expressed as

$$
\mathrm{TC}_{c 0}=\sum_{i}^{n}\left(\frac{1}{T_{i}}\left(F+f_{i}\right)+\frac{T_{i}}{2} D_{i} H_{c i}\right) .
$$

Since the manufacturers adopt a "make-to-order" policy, the total cost for each manufacturer per year is

$$
\begin{array}{r}
\mathrm{TC}_{s i 0}=\frac{S_{i}}{T_{i} k_{i}}+\frac{T_{i} D_{i} H_{s i}}{2}\left[\frac{2 D_{i}}{P_{i}}-1+\left(1-\frac{D_{i}}{P_{i}}\right) k_{i}\right] \\
i=1,2, \ldots n
\end{array}
$$

Then the total cost per year incurred to the retailer and the manufacturers for independent replenishing $n$ materials can be derived as

$$
\begin{aligned}
\mathrm{JTC}_{0}\left(k_{i}, T_{i}\right) \\
=\mathrm{TC}_{c 0}+\sum_{i=1}^{n} \mathrm{TC}_{s i 0} \\
=\sum_{i=1}^{n}\left(\frac{F+f_{i}}{T_{i}}+\frac{D_{i} H_{c i} T_{i}}{2}\right) \\
+\sum_{i=1}^{n}\left\{\frac{S_{i}}{T_{i} k_{i}}+\frac{T_{i} D_{i} H_{s i}}{2}\left[\frac{2 D_{i}}{P_{i}}-1+\left(1-\frac{D_{i}}{P_{i}}\right) k_{i}\right]\right\} \\
=\sum_{i=1}^{n} \frac{1}{T_{i}}\left(F+f_{i}+\frac{S_{i}}{k_{i}}\right) \\
+\sum_{i=1}^{n} \frac{D_{i} T_{i}}{2}\left[H_{c i}+H_{s i}\left(\frac{2 D_{i}}{P_{i}}-1\right)+H_{s i}\left(1-\frac{D_{i}}{P_{i}}\right) k_{i}\right] .
\end{aligned}
$$

To simplify the expression, we denote $A_{i}=F+f_{i}, B_{i}=$ $D_{i}\left[H_{c i}+H_{s i}\left(2 D_{i} / P_{i}-1\right)\right]$. Then we can obtain the simplified formulation of $\mathrm{JTC}_{0}$ as follows:

$$
\begin{aligned}
\operatorname{JTC}_{0}\left(k_{i}, T_{i}\right)= & \sum_{i=1}^{n} \frac{1}{T_{i}}\left(A_{i}+\frac{S_{i}}{k_{i}}\right) \\
& +\sum_{i=1}^{n} \frac{T_{i}}{2}\left[B_{i}+D_{i} H_{s i}\left(1-\frac{D_{i}}{P_{i}}\right) k_{i}\right] .
\end{aligned}
$$

For a given set of $K=\left(k_{1}, k_{2}, \ldots, k_{n}\right)$, taking the first order of $\mathrm{JTC}_{0}$ with respect to $T_{i}$ we have

$$
\begin{aligned}
\frac{\partial \mathrm{JTC}_{0}\left(k_{i}, T_{i}\right)}{\partial T_{i}}= & -\frac{A_{i}+S_{i} / k_{i}}{T_{i}^{2}} \\
& +\frac{1}{2}\left[B_{i}+D_{i} H_{s i}\left(1-\frac{D_{i}}{P_{i}}\right) k_{i}\right] .
\end{aligned}
$$

Let $\partial \mathrm{JTC}_{0}\left(k_{i}, T_{i}\right) / \partial T_{i}=0$; the optimal $T_{i}^{*}$ must satisfy

$$
T_{i}^{*}\left(k_{i}\right)=\sqrt{\frac{2\left(A_{i}+\left(S_{i} / k_{i}\right)\right)}{\left[B_{i}+D_{i} H_{s i}\left(1-D_{i} / P_{i}\right) k_{i}\right]}} .
$$

Since $\partial^{2} \mathrm{JTC}_{0}\left(k_{i}, T_{i}\right) / \partial T_{i}^{2}=2\left(A_{i}+S_{i} / k_{i}\right) / T_{i}^{3}>0, T_{i}^{*}$ can be determined uniquely by (6) after $k_{i}$ are given.

Substitute (6) into (4); the optimal $\mathrm{JTC}_{0}$ can be obtained by

$$
\begin{aligned}
\mathrm{JTC}_{0}^{*}\left(k_{i}\right) \\
\quad=\sum_{i=1}^{n} \sqrt{2\left(A_{i}+\frac{S_{i}}{k_{i}}\right)\left[B_{i}+D_{i} H_{s i}\left(1-\frac{D_{i}}{P_{i}}\right) k_{i}\right]} .
\end{aligned}
$$

2.3. Formulation of the JR-CC Model. The objective of the JRCC model is to minimize the total cost in this two-echelon supply chain. It involves determining a basic replenishment cycle time $T$ and the replenishment interval $k_{i} T$ for item $i$, where $k_{i}$ is an integral number. Then the total cost per year for the retailer is

$$
\mathrm{TC}_{c}=\frac{1}{T}\left(F+\sum_{i=1}^{n} f_{i}\right)+\frac{T}{2} \sum_{i=1}^{n} D_{i} H_{c i} .
$$
is

The total cost of material $i$ per year for the manufacturers

$$
\mathrm{TC}_{s i}=\frac{S_{i}}{k_{i} T}+\frac{T D_{i} H_{s i}}{2}\left[\frac{2 D_{i}}{P_{i}}-1+\left(1-\frac{D_{i}}{P_{i}}\right) k_{i}\right]
$$


Then the total cost per year incurred to the retailer and the manufacturers for joint replenishing $n$ materials can be derived as

$$
\begin{aligned}
\operatorname{JTC}\left(k_{i}, T\right)= & \mathrm{TC}_{c}+\sum_{i=1}^{n} \mathrm{TC}_{s i} \\
= & \frac{1}{T}\left(F+\sum_{i=1}^{n} f_{i}+\sum_{i=1}^{n} \frac{S_{i}}{k_{i}}\right) \\
& +\frac{T}{2}\left[\sum_{i=1}^{n} D_{i}\left(H_{c i}+H_{s i}\left(\frac{2 D_{i}}{P_{i}}-1\right)\right)\right. \\
= & \frac{1}{T}\left(A A+\sum_{i=1}^{n} \frac{S_{i}}{k_{i}}\right) \\
& +\frac{T}{2}\left[B B+\sum_{i=1}^{n} D_{i} H_{s i}\left(\left(1-\frac{D_{i}}{P_{i}}\right) k_{i}\right)\right]
\end{aligned}
$$

where $A A=F+\sum_{i=1}^{n} f_{i}, B B=\sum_{i=1}^{n} D_{i}\left(H_{c i}+H_{s i}\left(2 D_{i} / P_{i}-\right.\right.$ $1))$. Since the formulation of JTC has the similar structure of $\mathrm{JTC}_{0}$, for any fixed $k_{i}$, the corresponding optimal common shipment cycle time $T^{*}(K)$ is given by

$$
T^{*}\left(k_{i}\right)=\sqrt{\frac{2\left(A A+\sum_{i=1}^{n} S_{i} / k_{i}\right)}{B B+\sum_{i=1}^{n} D_{i} H_{s i}\left(1-D_{i} / P_{i}\right) k_{i}}} .
$$

Then the minimal total cost of the supply chain under channel cooperation is

$$
\begin{aligned}
& \operatorname{JTC}^{*}\left(k_{i}\right) \\
& =\sqrt{2\left(A A+\sum_{i=1}^{n} \frac{S_{i}}{k_{i}}\right)\left[B B+\sum_{i=1}^{n} D_{i} H_{s i}\left(\left(1-\frac{D_{i}}{P_{i}}\right) k_{i}\right)\right]} .
\end{aligned}
$$

Obviously, both the JR-CC model and IR model have similar function structures. Hsu [14] proposed a heuristic for solving this model and obtaining the optimal solution. However, the heuristic is not universal and relatively complex for decision-makers. In addition, the JR-CC model is extended considering the restriction of coordination cost in the sensitivity analysis. So the heuristic cannot solve this model with a cost constraint. Therefore, in order to analyze model more effectively, a DE-based solution is provided.

\section{The Improved DE (IDE) for the JR-CC Model}

\subsection{The Classical DE and Improvement}

3.1.1. The Classical DE. Unlike other evolutionary algorithms, $\mathrm{DE}$ does not make use of some probability distribution function in order to introduce variations into the population (Wang et al. [7]; Salman et al. [31]). DE initializes population randomly by uniform distribution over search space. Each individual is called target vector. A new vector is created by adding the weighted difference of two random vectors to target vector. Then a trail vector is generated by mixing the mutated vectors with the target vectors according to selected rules. Finally, a one-to-one competition selects the best one between the trail vector and the corresponding target vector according to its fitness (Wang et al. [32]; Brest et al. [33]). Three operations of the classical DE are introduced as follows.

Mutation. For each target vector $x_{t}^{(G)}$ of generation $G$, a mutated vector $v_{t}^{(G+1)}$ is created according to the following:

$$
v_{t}^{(G+1)}=x_{r 1}^{(G)}+F F^{*}\left(x_{r 2}^{(G)}-x_{r 3}^{(G)}\right),
$$

where $G$ presents the present generation; $F F \in[0,2]$ is a mutation factor used to control the amplification of the differential variation; $r_{1}, r_{2}$, and $r_{3}$ are three distinct random numbers and none of them coincides with the current target individual $t\left(r_{1} \neq r_{2} \neq r_{3} \neq t\right)$.

Crossover. The trail vector can be obtained using the following rules:

$$
u_{t j}^{(G+1)}= \begin{cases}v_{t j}^{(G+1)} & \text { if }(\text { rand } \leq C R) \text { or } j=\operatorname{rnbr}(t), \\ x_{t j}^{(G)} & \text { if }(\text { rand }>C R) \text { and } j \neq \operatorname{rnbr}(t),\end{cases}
$$

where $\operatorname{rand}(j)$ is a uniformly distributed random number in range $(0,1) ; \operatorname{rnbr}(t)$ is a randomly chosen integer in the $\operatorname{set}\left\{1,2, \ldots, N_{p}\right\}$ which ensures the trail vector gets at least one parameter from the mutated vector; $C R \in[0,1]$ is a crossover constant.

Selection. The selection mechanism adopts one-to-one competition greedy strategy to decide which vector (trail vector $u_{t}^{(G+1)}$ and target vector $\left.x_{t}^{(G)}\right)$ is chosen into the next generation. That is to say, $u_{t}^{(G+1)}$ replaces $x_{t}^{(G)}$ only if its fitness (i.e., the value of objective function) is better than the fitness of $x_{t}^{(G)}$.

3.1.2. The Improved $D E$ (IDE). In order to improve $\mathrm{DE}$, an adaptive parameter $F$ is adopted and a new selection method based on DE and genetic algorithm (GA) is utilized.

Adaptive Mutation Factor. In mutation, parameter FF plays an important role. FFaffects the speed of convergence anddecides the search range. Usually, optimization algorithms favor global search at the early stage for exploring feasible domain and local search at the latter stage for accelerating convergence. Based on above features, a parameter $F F$ is defined as follows:

$$
F F=F F_{\min }+\left(F F_{\max }-F F_{\min }\right) * e^{1-(\operatorname{Gen} M /(\operatorname{Gen} M-G+1))},
$$

where $F F_{\min }$ is the lower bound of $F F ; F F_{\max }$ is the upper bound of $F F$; GenM is maximum evolution generation; $G$ 
presents the evolution generation. So FF can adjust its size with the change of the iterations. The larger mutation factor ensures greater diversity of population at the early stage, and the smaller mutation factor reserves excellent individual at the latter stage.

Combined Selection Operation of GA. The truncation selection method of GA is adopted. The trial solution is not compared with its parent but is kept in the trial set. When all individuals in the population generate their trial vector, a new population size of $2 N_{P}$ is integrated by combining trial set and parent set. Then their fitness values are sorted in ascending order and the previous $50 \%$ vectors are truncated for the next generation population of the algorithm to ensure the excellent solution.

3.1.3. An Example for IDE. Here an example is given to illustrate the procedure of the proposed DE algorithm. For current number of iteration $G$ and target vector $x_{1}^{(G)}$, suppose random generated numbers $r_{1}, r_{2}$, and $r_{3}$ are 23,40 , and $N_{p}$, respectively:

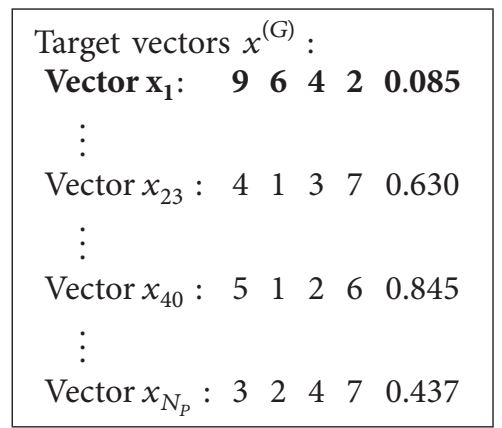

Mutation. If the calculated $F F=0.6$, then the mutated vector $v_{1}^{(G+1)}$ can be obtained by (13):

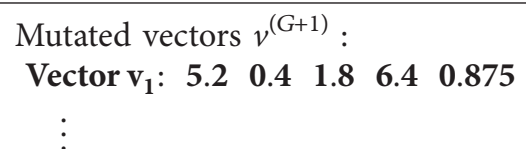

Crossover. If $C R=0.3 \operatorname{rnbr}(t)=3$ (here $t=1$ ), and vector rand $=(0.1,0.4,0.5,0.2,0.6)$, the trial vector can be obtained from (14):

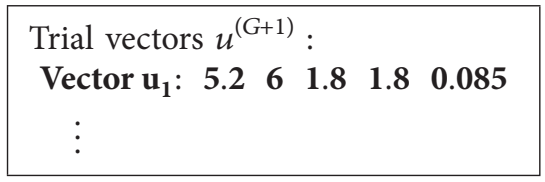

Selection. In this operation, the superior $N_{p}$ individuals are chosen to next generation from the set of $\left\{x^{(G)}, u^{(G+1)}\right\}$ according to the improved selection scheme.

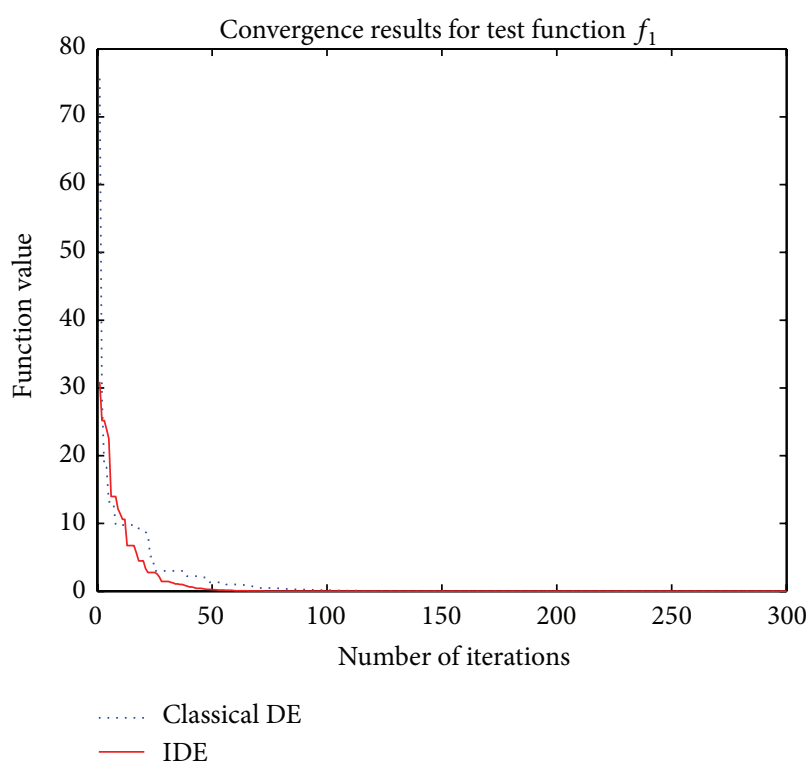

FIGURE 1: Convergence results of $f_{1}$.

\subsection{Comparative Study of the Classical DE and IDE by Benchmark Functions}

3.2.1. Test Functions. In order to verify the performance of improved DE, four benchmark functions used by Brest et al. [33] are utilized to test the performance of IDE. To assure a relatively fair comparison, the functions are selected according to their different property. Function $f_{1}$ is a unimodal function, $f_{2}$ is a step function, and $f_{3}-f_{4}$ are multimodal functions which appear to be the most difficult class of problems for many intelligent algorithms. Four benchmark functions are given in Table 1.

3.2.2. Comparative Results. The comparative study involves the speed of convergence and the ability to obtain optimal solution. Two algorithms including the classical DE and IDE are compared. In all cases, the number of populations $\left(N_{p}\right)$ is 200; maximum number of iterations (GenM) is 300 and 500 for $L=10$ and $L=30$, respectively. For the classical $\mathrm{DE}$ and IDE, crossover rate $C R$ is 0.3 . The mutation rate $F F$ for the classical DE is $0.6 ; F F_{\text {min }}$ is 0.2 and $F F_{\text {max }}$ is 1.2 for IDE. The decision for using these values is based on the experiences from literatures (Wang et al. [17]; Storn and Price [28]; Brest et al. [33]). Based on Matlab 7.0, all experiments are programmed on a computer (CPU: Intel Core 2 Duo T5870 @ 2.00 GHz 2.00 GHz; RAM: $992 \mathrm{MB}$; OS: Microsoft Windows $\mathrm{XP}$ ). The convergence results are presented in Figures 1, 2, 3, and 4.

Figures 1, 2, 3, and 4 demonstrate that IDE converges faster than classical DE in all cases, particularly when functions are difficult to converge such as $f_{3}$. Table 2 summarizes values averaged over 50 independent runs for the average $f_{\min }$ and standard deviation $f_{\text {min }}$ of each algorithm for different test functions. The average CPU time and standard derivation of CPU time are reported in Table 3. 
TABLE 1: Four benchmark functions.

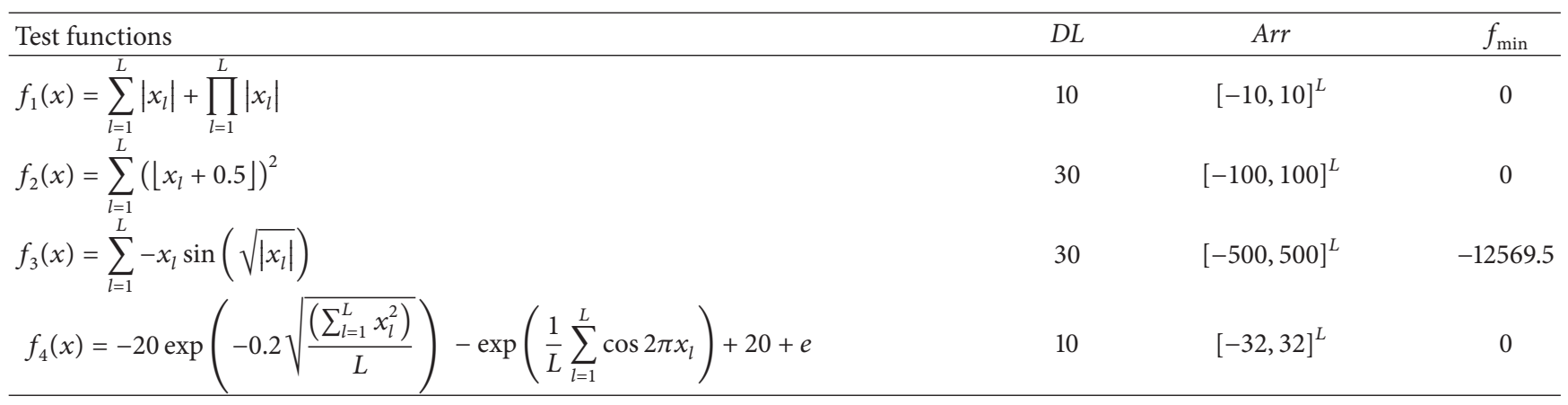

$D L$ denotes the dimensionality of the test problem, Arr denotes the ranges of the variables, and $f_{\min }$ is a function value of the global optimum.

TABLE 2: Results of two different algorithms under four test functions.

\begin{tabular}{lcccc}
\hline Test function & IDE & & \multicolumn{2}{c}{ Classical DE } \\
& Mean $f_{\min }$ & Std. dev. $f_{\text {min }}$ & Mean $f_{\text {min }}$ & Std. dev. $f_{\text {min }}$ \\
\hline$f_{1}$ & $2.6794 E-012$ & $9.1466 E-013$ & $6.0456 E-006$ & $1.4096 E-006$ \\
$f_{2}$ & 0 & 0 & -8473.9 & 2.7080 \\
$f_{3}$ & -12569.5 & 0 & $4.4122 E-005$ & 167.4174 \\
$f_{4}$ & $3.4866 E-010$ & $1.1557 E-010$ & $7.7586 E-006$ \\
\hline
\end{tabular}

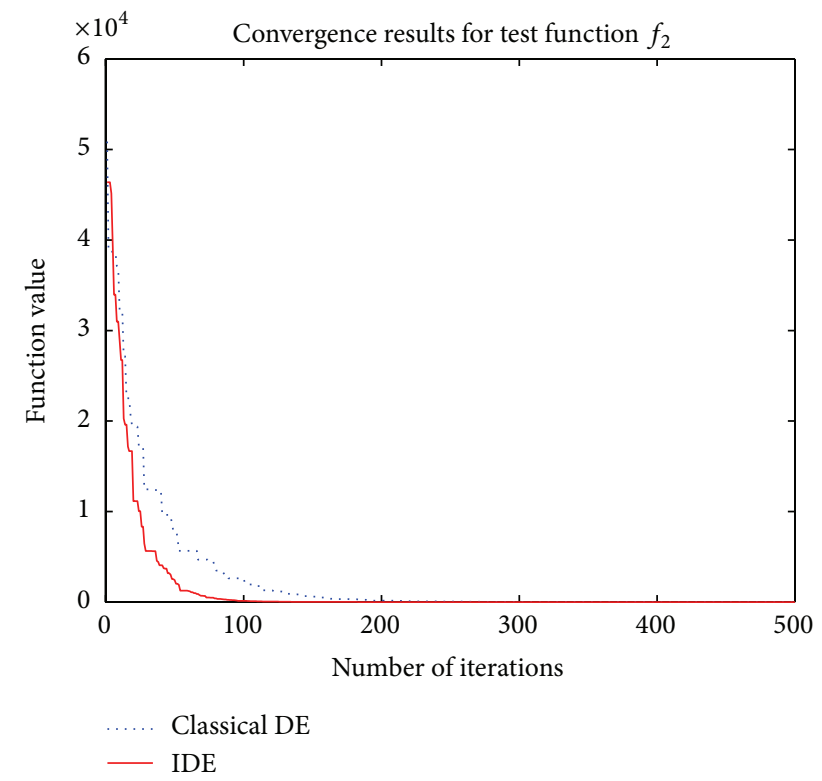

FIGURE 2: Convergence results of $f_{2}$.

Table 2 illustrates that IDE is more effective to obtain optimal solutions, especially for $f_{2}$ and $f_{3}$. Moreover, the fluctuation from average $f_{\min }$ of IDE is much smaller than that of classical DE, which also indicates the stability of IDE. Tables 2 and 3 show IDE always can find better solutions than DE faster.

3.3. IDE-Based Procedure for JR-CC Model. Considering that IR model and JR-CC model have similar property, for a given set of $K$, we can obtain the optimal $T_{i}$ according to (6), $T$

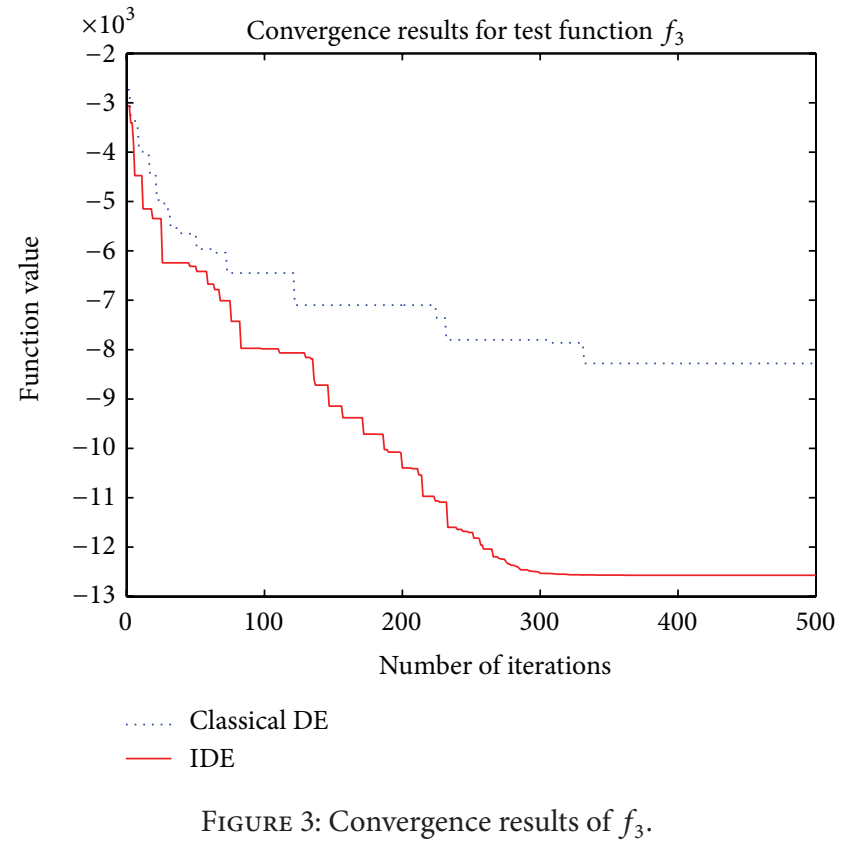

according to (11), and the optimal function value, that is, (7) for the IR model and (12) for the JR-CC model. Thus the procedure for two models is similar, and in the following the JR-CC model is used as an example to introduce the procedure.

Step 1 (initialization). Set lower and upper bound of mutation factor $F F$, crossover factor $C R$, and population scale $N_{P}$. Give the maximum number of iterations GenM and the dimension of each individual $D L$. Set the lower bound and the upper bound of $k_{i}$, respectively (donated as $k_{i}^{L B}, k_{i}^{U B}$ ). Note that $k_{i}$ 


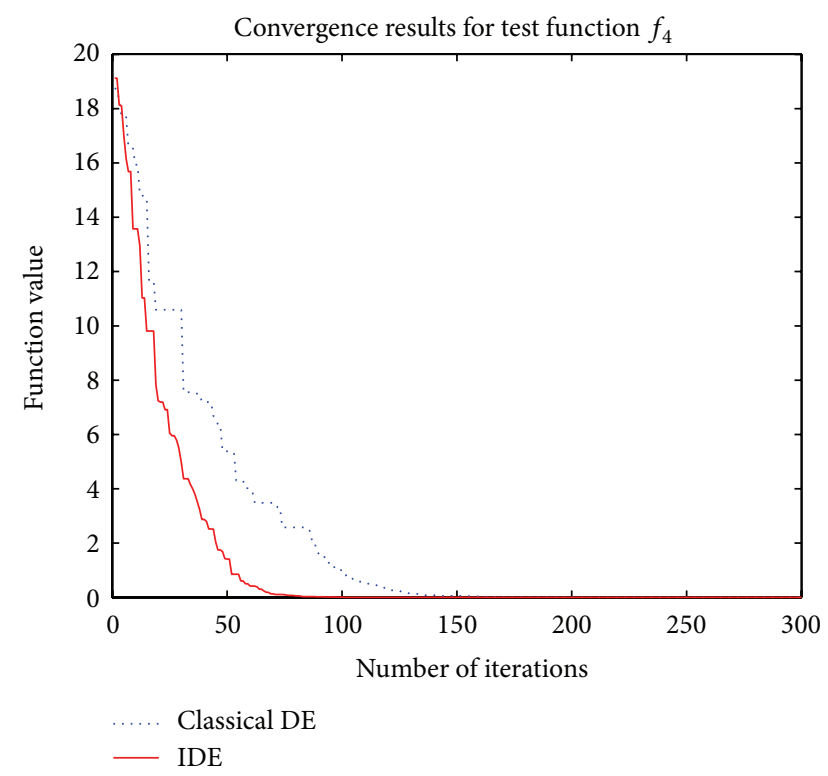

FIGURE 4: Convergence results of $f_{4}$.

TABLE 3: CPU time (s) of two different algorithms under four test functions.

\begin{tabular}{lcccc}
\hline \multirow{2}{*}{$\begin{array}{l}\text { Test } \\
\text { function }\end{array}$} & Mean & Std. dev. & \multicolumn{2}{c}{ Classical DE } \\
& CPU time & CPU time & CPU time & Std. dev. \\
CPU time
\end{tabular}

are integers so $k_{i}^{L B}$ is obviously 1 . According to the similar experience of Wang et al. [7] and Wang et al. [17], $k_{i}^{U B}$ is set sufficiently large to guarantee that optimal solution does not escape, such as 100. Generate the initial population $x^{(0)}=$ $\left(x_{1}^{(0)}, x_{2}^{(0)}, \ldots, x_{N_{P}}^{(0)}\right)$ randomly between $k_{i}^{L B}$ and $k_{i}^{U B}$.

Step 2. While stopping criterion is not met $(G<G e n M)$, go to Step 3; else go to Step 4.

Step 3. For each individual $x_{t}^{(G)}=\left(x_{t 1}^{(G)}, x_{t 2}^{(G)}, \ldots, x_{t D}^{(G)}\right), t=$ $1,2, \ldots, N_{P}$.

Step 3.1 (mutation). Choose three vectors $x_{r 1}^{(G)}, x_{r 2}^{(G)}, x_{r 3}^{(G)}$ randomly from the current population. Generate the mutated vector $v_{t}^{(G)}$ by (13).

Step 3.2 (crossover). Generate the trail vector $u_{t}^{(G+1)}$ by (14).

Step 3.3 (selection). Sort the new population size of $2 N_{p}$ with trial set and parent set by optimal objective function value, and then truncate the previous $50 \%$ into next generation population $x^{(G+1)}$.

Step 4. Output the best results.

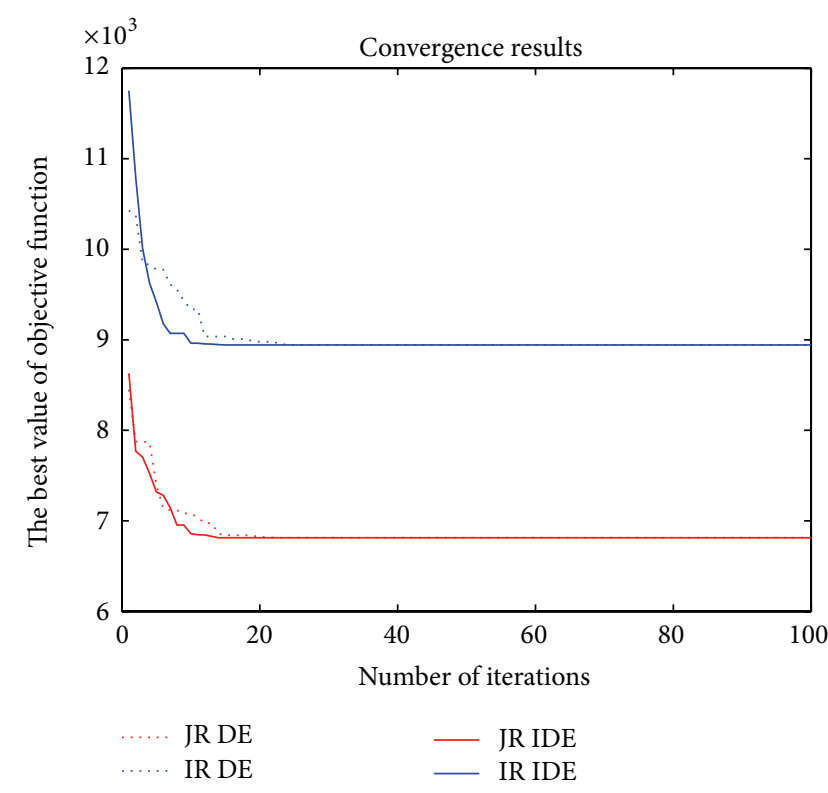

Figure 5: The convergence curves of DE and IDE.

\section{Numerical Examples and Sensitivity Analysis}

In this section, three numerical examples are presented. To verify the accuracy of IDE and the classical DE for the proposed models, a comparative example of Hsu [14] is conducted firstly. In order to further test the performance of the proposed algorithm for the practical problem, an extended example of the larger scale is presented in Section 4.2. For the analysis of the impact of different parameters of the models on the policy, a sensitivity analysis is designed in Section 4.3.

4.1. Comparative Example of Hsu [14] and Analysis. For the sake of analysis and verifying the accuracy of the IDE and the classical DE, the same data as Hsu [14] is adopted. The related parameters are reported in Table 4.

Considering the advice of Neri and Tirronen [34] and Wang et al. [35], the following parameters are set: $F F_{\min }=$ $0.2, F F_{\max }=1.2, F F=0.6, C R=0.3$, and $N_{p}=5 D L$. GenM is set to 100 and the dimension of each individual $D L$ is as the same with the number of materials $n$. For a better comparison, the cost of each entity and the total cost in the supply chain are calculated. Moreover, the differences of each cost between two models are shown in Table 5. Figure 5 gives the convergence curves of the DE and IDE.

Table 5 shows the optimal values of decision variables $K$ of IR model and JR-CC model using IDE and the classic DE are the same with the heuristic of Hsu [14] and the little difference of cost of the supply chain between Hsu [14] and ours due to the number of decimal digits of decision variables $T_{i}$. Compared with the IR policy, the total cost of the system is reduced by $23.83 \%$ using the JR-CC policy. In particular, the cost of the retailer is cut down by $31.28 \%$. This indicates that the JR-CC model can reduce supply chain costs obviously 
TABle 4: Parameter settings.

\begin{tabular}{lccc}
\hline & Production rate $P_{i}$ & Setup cost $S_{i}$ & Carrying cost rate $H_{s i}$ \\
\hline Manufacturer 1 & 18000 & 45 & 2 \\
Manufacturer 2 & 18000 & 45 & 1 \\
Manufacturer 3 & 18000 & 45 & 1 \\
\hline \multicolumn{4}{c}{} \\
Retailer & 10000 & Minor ordering cost $f_{i}$ & Carrying cost rate $H_{c i}$ \\
& 12000 & 8 & 8 \\
& 9000 & 5 & 4 \\
\end{tabular}

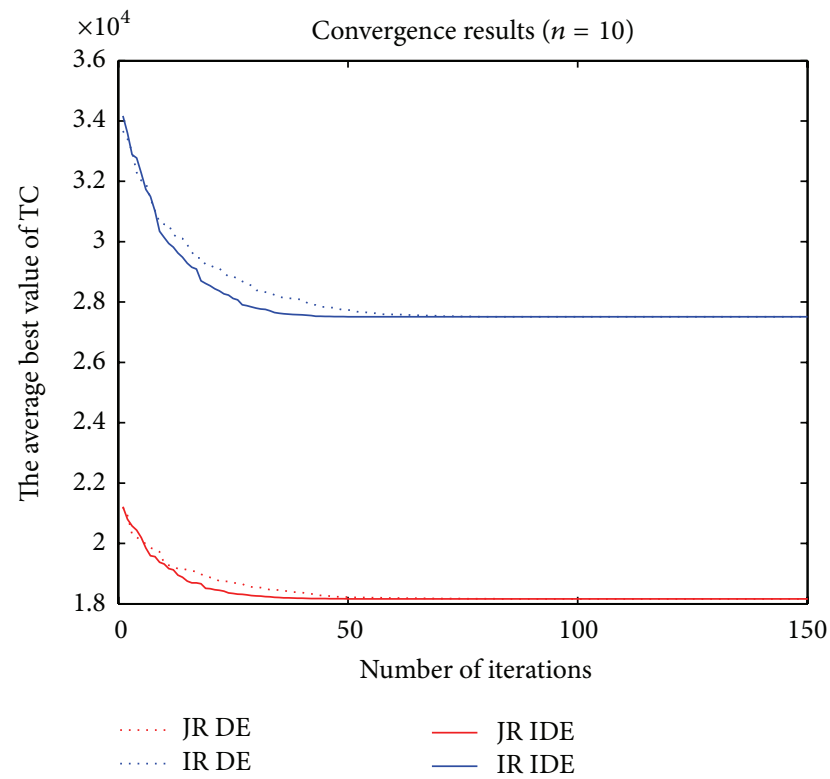

FIGURE 6: The average convergence curves $(n=10)$.

and enhance supply chain's efficiency. Furthermore, the convergence curves in Figure 5 demonstrate IDE can converge to the optimal results faster than DE.

4.2. An Extended Example with Larger Scale. Since the problem scale of Hsu [14] is too small, an extended example with 10, 30, and 50 materials is designed in this section to verify the performance of the proposed algorithm further. The input parameters are generated from Table 6 .

The parameters $D_{i}, f_{i}, H_{c i}$, and $H_{s i}$ are generated randomly from the given ranges in Table 6. The algorithm's parameters $F F_{\text {min }}, F F_{\text {max }}, F F, C R$, and $N_{p}$ are set the same as in Section 4.1; the maximum iteration number GenM is set to 150,300 , and 600 , respectively, for $n=10, n=30$, and $n=50$. Each scale is running 20 times. The results are reported in Table 7 , and the average convergence curves are listed in Figures 6, 7, and 8.

The results shown in Table 7 and the curves in Figures 6 , 7 , and 8 show the following interesting conclusions.

(1) For different scales, IDE can always converge to the optimal solution with the convergence rate of $100 \%$.

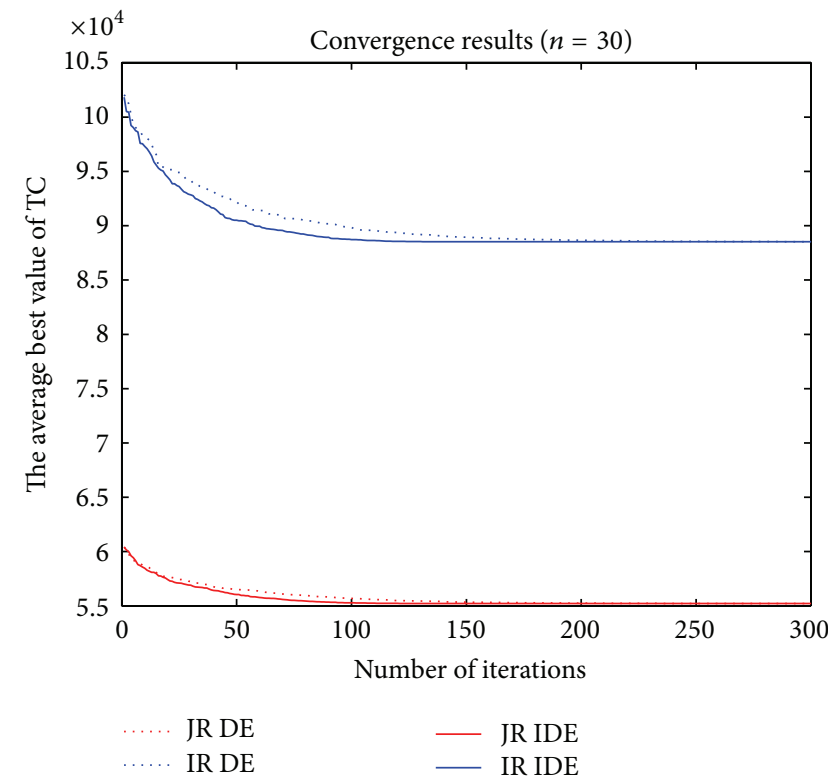

Figure 7: The average convergence curves $(n=30)$.

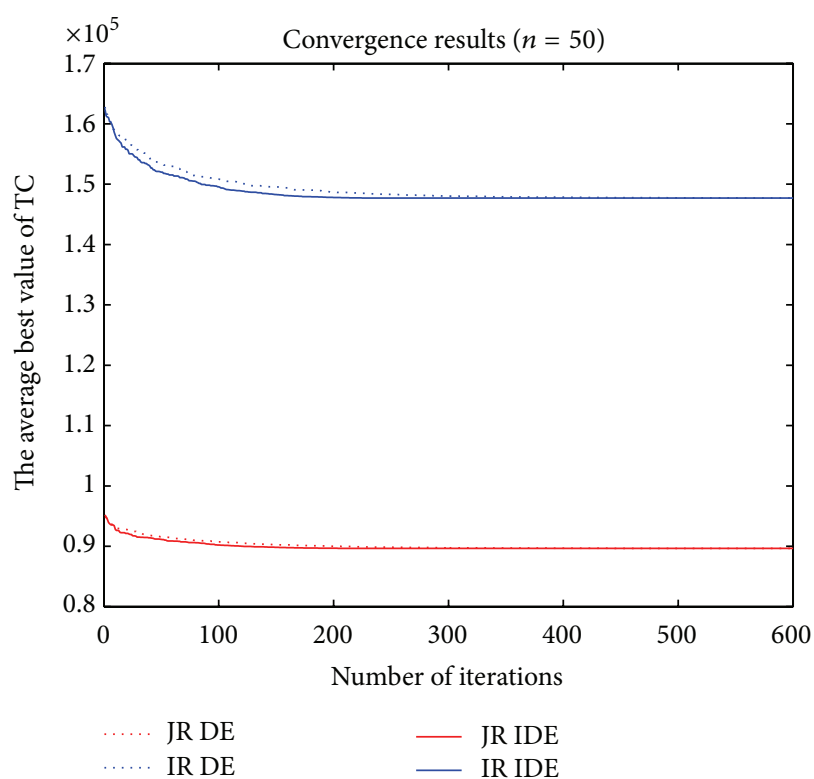

Figure 8: The average convergence curves $(n=50)$. 
TABLE 5: Comparison between independent replenishment and JR-CC policy.

\begin{tabular}{|c|c|c|c|c|c|}
\hline & \multicolumn{2}{|c|}{ IR } & \multicolumn{2}{|c|}{ JR-CC } & \multirow{2}{*}{ Cost reduction } \\
\hline & $\mathrm{DE}$ (IDE) & Hsu [14] & $\mathrm{DE}$ (IDE) & Hsu [14] & \\
\hline Cost of retailer & 6700.3 & & 4604.4 & & $31.28 \%$ \\
\hline Cost of manufacturer 1 & 931.4354 & & 923.6882 & & $0.83 \%$ \\
\hline Cost of manufacturer 2 & 673.8471 & & 647.3242 & & $3.94 \%$ \\
\hline Cost of manufacturer 3 & 637.0856 & & 636.6200 & & $0.07 \%$ \\
\hline \multirow[t]{2}{*}{ Cost of the supply chain } & $8942.7^{*}$ & $8942.64^{*}$ & 6812.0 & 6811.99 & $23.83 \%$ \\
\hline & $T_{1}=0.0312$ & $T_{1}=0.031$ & & & \\
\hline \multirow[t]{3}{*}{ Shipment cycle time $T$} & $T_{2}=0.0369$ & $T_{2}=0.037$ & $T=0.023$ & $T=0.023$ & \\
\hline & $T_{3}=0.0337$ & $T_{3}=0.034$ & & & \\
\hline & $k_{1}=3$ & $k_{1}=3$ & $k_{1}=4$ & $k_{1}=4$ & \\
\hline \multirow[t]{2}{*}{$K^{*}$} & $k_{2}=4$ & $k_{2}=4$ & $k_{2}=7$ & $k_{2}=7$ & \\
\hline & $k_{3}=4$ & $k_{3}=4$ & $k_{3}=6$ & $k_{3}=6$ & \\
\hline
\end{tabular}

${ }^{*}$ Represents the meaning of "the optimal", which had been hinted in Section 2.2 and Section 2.3.

TABLE 6: Parameters for the extended problem.

\begin{tabular}{llc}
\hline Parameters & & Value \\
\hline$n$ & Number of materials & $10,30,50$ \\
$D_{i}, i=1,2, \ldots, n$ & Demand rate & $U[6000,15000]$ \\
$P_{i}, i=1,2, \ldots, n$ & Production rate & 18000 \\
$S_{i}, i=1,2, \ldots, n$ & Setup cost of material $i$ for the manufacturers & 45 \\
$F$ & Major ordering cost for the retailer & 30 \\
$f_{i}, i=1,2, \ldots, n$ & Minor ordering cost of material $i$ for the retailer & $U[4,12]$ \\
$H_{c i}, i=1,2, \ldots, n$ & Carrying cost rate of material $i$ for the retailer & $U[4,8]$ \\
$H_{s i}, i=1,2, \ldots, n$ & Carrying cost rate of material $i$ for the manufacturers & $U[1,2]$ \\
\hline
\end{tabular}

(2) The CPU time to obtain the optimal solution of IDE is always faster than DE.

(3) The convergence speed of IDE is faster than DE too. So, whatever in robustness, computing time, or convergence speed, IDE is always better than DE. IDE is a better candidate algorithm for the JR-CC model and IR model.

4.3. Sensitivity Analysis. Since the fundamental of joint replenishment is sharing the major ordering cost $F$ to achieve cost saving, and the value of coordination cost $L$ to implement JR policy has a direct impact on the selection of the policies, we mainly examine the impact of these two parameters on the JR-CC model and IR model. Based on the numerical examples above, IDE is adopted for the sensitivity analysis.

4.3.1. The Impact of Misestimates of Fon the Cost Reduction of the Supply Chain. The results obtained by IDE with $F$ varying from 0 to $2 F$ based on the basic data in Table 4 are shown in Table 8 .

Table 8 shows the cost reduction of the whole supply chain and almost every entity is increasing when the value of $F$ becomes larger. The retailer, especially, has more cost savings. In order to observe this trend more clearly, Figure 9 is provided using the data of Table 8 .

It is obvious that the changing of major ordering cost $F$ only causes a little increase in all manufacturers (less than $7 \%)$, but very tremendous in the retailer and the whole supply chain (more than 17\%). This indicates that major ordering cost causes more influence on the retailer than the manufacturers. In other words, the retailer has more willingness to adopt the joint replenishment and channel coordination policy because of the difference of the cost reduction value. But the manufacturers may be reluctant to accept the policy. We also find that if the value of $F$ is too low, the retailer also has no interest in JR-CC policy because the cost reduction is too little.

4.3.2. The Impact of the Coordination Cost on the Cost Reduction of the Supply Chain. When the retailer combines the manufacturers to adopt the JR-CC policy, the relevant responsible staff and certain resources including telephone communication, mail communication, and business trips should be needed. Since the cost of these resources is hard to be estimated or is small, almost theoretical researches ignore the coordination cost in the existing JR-CC model. In order to be more practical, here we will consider a new parameter $L$ representing the coordination cost. From Table 5 we know 
TABLE 7: The results of different scales under different algorithms.

\begin{tabular}{|c|c|c|c|c|c|c|c|c|}
\hline \multirow{2}{*}{$\begin{array}{l}\text { Problem } \\
\text { scale }\end{array}$} & \multirow[b]{2}{*}{ Algorithm } & \multicolumn{3}{|c|}{ IR } & \multicolumn{3}{|c|}{ JR-CC } & \multirow[b]{2}{*}{ Cost reduction } \\
\hline & & $\mathrm{JTC}_{0}^{*}$ & $\begin{array}{c}\text { The number to } \\
\text { obtain } \mathrm{JTC}_{0}^{*}\end{array}$ & $\begin{array}{c}\text { Average CPU } \\
\text { time }(\mathrm{s})\end{array}$ & $\mathrm{JTC}^{*}$ & $\begin{array}{c}\text { The number to } \\
\text { obtain JTC }\end{array}$ & $\begin{array}{c}\text { Average CPU } \\
\text { time }(\mathrm{s})\end{array}$ & \\
\hline \multirow{2}{*}{$n=10$} & $\mathrm{DE}$ & $2.7511 E 4$ & 20 & 0.7450 & $1.8161 E 4$ & 20 & 0.7472 & \multirow{2}{*}{$33.99 \%$} \\
\hline & IDE & $2.7511 E 4$ & 20 & 0.4037 & $1.8161 E 4$ & 20 & 0.4062 & \\
\hline \multirow{2}{*}{$n=30$} & $\mathrm{DE}$ & $8.8528 E 4$ & 9 & 7.5445 & $5.5214 E 4$ & 20 & 7.3905 & \multirow{2}{*}{$37.63 \%$} \\
\hline & IDE & $8.8528 E 4$ & 20 & 4.1093 & $5.5214 E 4$ & 20 & 3.9868 & \\
\hline \multirow{2}{*}{$n=50$} & $\mathrm{DE}$ & $1.4770 E 5$ & 0 & 14.5153 & $8.9647 E 4$ & 0 & 13.1147 & \multirow{2}{*}{$39.30 \%$} \\
\hline & IDE & $1.4769 E 5$ & 20 & 7.8123 & $8.9644 E 4$ & 20 & 7.6407 & \\
\hline
\end{tabular}

${ }^{*}$ Represents the meaning of "the optimal", which had been hinted in Section 2.2 and Section 2.3.

TABLE 8: Sensitivity analysis of major ordering cost $F$.

\begin{tabular}{|c|c|c|c|c|c|c|}
\hline Parameter variable & Model & Retailer & Manufacturer 1 & Manufacturer 2 & Manufacturer 3 & Supply chain \\
\hline \multirow{3}{*}{$\begin{array}{l}F \\
(1-100 \%)\end{array}$} & IR & 3025.2 & 910.2592 & 627.5493 & 636.8673 & 5199.88 \\
\hline & JR-CC & 3034.3 & 911.1031 & 629.5798 & 636.953 & 5211.94 \\
\hline & Cost reduction & $-0.30 \%$ & $-0.09 \%$ & $-0.32 \%$ & $-0.01 \%$ & $-0.23 \%$ \\
\hline \multirow{3}{*}{$\begin{array}{l}F \\
(1-50 \%)\end{array}$} & IR & 5201.9 & 922.0195 & 657.6866 & 637.2893 & 7418.90 \\
\hline & JR-CC & 3898.8 & 916.5907 & 639.1153 & 636.9343 & 6091.44 \\
\hline & Cost reduction & $25.05 \%$ & $0.59 \%$ & $2.82 \%$ & $0.06 \%$ & $17.89 \%$ \\
\hline \multirow{3}{*}{$\begin{array}{l}F \\
(1-25 \%)\end{array}$} & IR & 6001.9 & 925.4559 & 664.7484 & 637.0900 & 8229.19 \\
\hline & JR-CC & 4267.3 & 918.1299 & 641.9134 & 636.7032 & 6464.05 \\
\hline & Cost reduction & $28.90 \%$ & $0.79 \%$ & $3.44 \%$ & $0.06 \%$ & $21.45 \%$ \\
\hline \multirow{3}{*}{$F$} & IR & 6700.3 & 931.4354 & 673.8471 & 637.0856 & 8944.65 \\
\hline & JR-CC & 4604.4 & 923.6882 & 647.3242 & 636.62 & 6812.03 \\
\hline & Cost reduction & $31.28 \%$ & $0.83 \%$ & $3.94 \%$ & $0.07 \%$ & $23.83 \%$ \\
\hline \multirow{3}{*}{$\begin{array}{l}F \\
(1+25 \%)\end{array}$} & IR & 7336.9 & 931.4831 & 680.5486 & 636.5465 & 9585.48 \\
\hline & JR-CC & 4919.6 & 921.9574 & 648.8993 & 636.7276 & 7127.18 \\
\hline & Cost reduction & $32.95 \%$ & $1.02 \%$ & $4.65 \%$ & $-0.03 \%$ & $25.65 \%$ \\
\hline \multirow{3}{*}{$\begin{array}{l}F \\
(1+50 \%)\end{array}$} & IR & 7919.1 & 934.9171 & 693.3752 & 638.5272 & 10185.92 \\
\hline & JR-CC & 5217.3 & 922.6537 & 650.8138 & 638.1269 & 7428.89 \\
\hline & Cost reduction & $34.12 \%$ & $1.31 \%$ & $6.14 \%$ & $0.06 \%$ & $27.07 \%$ \\
\hline \multirow{3}{*}{$\begin{array}{l}F \\
(1+100 \%)\end{array}$} & IR & 8978.5 & 948.1541 & 700.000 & 637.3805 & 11264.03 \\
\hline & JR-CC & 5763.7 & 931.1428 & 657.4859 & 636.4094 & 7988.74 \\
\hline & Cost reduction & $35.81 \%$ & $1.79 \%$ & $6.07 \%$ & $0.15 \%$ & $29.08 \%$ \\
\hline
\end{tabular}

the cost of retailer is 6700.3 under IR policy, and then the JRCC model for the retailer will contain a constraint which can be derived as

$$
T C_{c}=\frac{1}{T}\left(F+\sum_{i=1}^{n} f_{i}\right)+\frac{T}{2} \sum_{i=1}^{n} D_{i} H_{c i}<6700.3-L
$$

when $T=\sqrt{2\left(F+\sum_{i=1}^{n} f_{i}\right) / \sum_{i=1}^{n} D_{i} H_{c i}}$; the left of constraint has minimal value $\sqrt{2\left(F+\sum_{i=1}^{n} f_{i}\right)\left(\sum_{i=1}^{n} D_{i} H_{c i}\right)}=4604.3$. Then we can calculate $L$ less than 2094.5 if the retailer implements the JR-CC policy. Since $L$ is only concluded in the private cost calculation of the retailer, it has no evident influence on the cost of manufacturers. Next, the impact of misestimates of $L$ on the cost reduction of the retailer who advocates the JR-CC policy is analyzed using the DE approach. The results are shown in Table 9.

Table 9 shows the cost reduction of the retailer is decreasing when $L$ is larger. When $L$ is small ( $L=10,50,100)$, the results change a little and the retailer can neglect it for the JRCC decision. In realistic circumstances, $L$ is not insignificant. But once $L$ becomes very large, it will have a huge impact on the results when $L=1500$ or $L=2000$.

\section{Conclusion and Future Research}

This paper is an interdisciplinary research of an operation management problem under supply chain environment and an intelligent optimization algorithm. We discussed a practical and useful JR-CC model and proposed an effective and 
TABLE 9: The sensitivity analysis of coordination $\operatorname{cost} L$ for the retailer.

\begin{tabular}{|c|c|c|c|c|c|c|c|c|}
\hline Model & $L=0$ & $L=10$ & $L=50$ & $L=100$ & $L=500$ & $L=1000$ & $L=1500$ & $L=2000$ \\
\hline IR & & & & & 00.3 & & & \\
\hline JR-CC & 4604.4 & 4614.4 & 4654.4 & 4704.4 & 5104.4 & 5604.4 & 6104.4 & 6604.4 \\
\hline Cost reduction & $31.28 \%$ & $31.13 \%$ & $30.53 \%$ & $29.79 \%$ & $23.82 \%$ & $16.36 \%$ & $8.90 \%$ & $1.43 \%$ \\
\hline
\end{tabular}

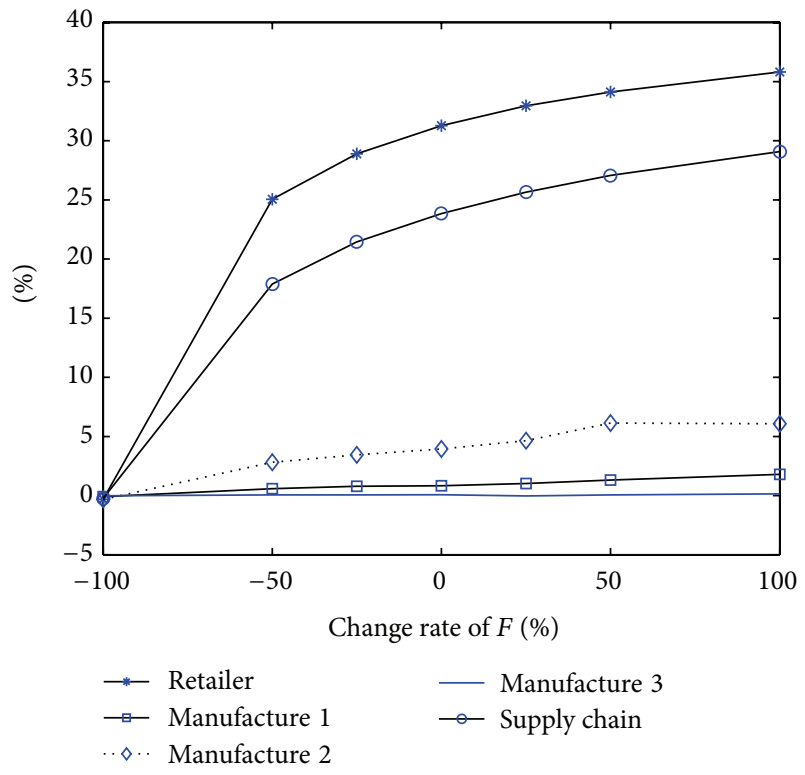

FIGURE 9: Impact of variation with $F$ on the cost reduction of the supply chain.

simple algorithm to handle this NP-hard problem. The main contributions are as follows.

(1) We consider the coordination cost when the retailer made JR-CC decisions while no existing works pay attention on it. Results of numerical examples show that the JR-CC policy can reduce total costs obviously and enhance the efficiency of a supply chain. But not all members in the supply chain can benefit a lot from this policy. The discussed JR-CC models can be applied in these industries, such as manufacturing and wholesale supplies under JIT purchasing environment.

(2) An IDE with an adaptive parameter FF is utilized based on the classic DE and GA. Results of benchmark functions and comparative numerical example verify the effectiveness of the proposed IDE. IDE is a good candidate for the JR-CC model.

(3) The impacts of misestimates of major ordering cost and the coordination cost on the cost reduction of the supply chain are analyzed, respectively. Corresponding managerial insights are given. Results of sensitivity analysis show the retailers have more willingness to adopt the JR-CC policy than the manufacturers because of the different cost savings.
However, the decision makers often have to face vague operational conditions. In this case, the fuzzy set theory is a useful approach to deal with this kind of problem [36]. In the future, integrated joint replenishment and channel coordination models under fuzzy environment can be developed. The DEs still can be used to provide a good solution to these problems.

\section{Conflict of Interests}

The authors declare that there is no conflict of interests regarding the publication of this paper.

\section{Acknowledgments}

The authors are very grateful for the constructive comments of editors and referees. This research is partially supported by National Natural Science Foundation of China (71371080; 71131004; 71101060), Humanities and Social Sciences Foundation of Chinese Ministry of Education (no. 11YJC630275), and Fundamental Research Funds for the Central Universities (HUST: 2014QN201).

\section{References}

[1] S. Axsäter and W.-F. Zhang, "Joint replenishment policy for multi-echelon inventory control," International Journal of Production Economics, vol. 59, no. 1, pp. 243-250, 1999.

[2] L. Wang, J. He, D. Wu, and Y.-R. Zeng, "A novel differential evolution algorithm for joint replenishment problem under interdependence and its application," International Journal of Production Economics, vol. 135, no. 1, pp. 190-198, 2012.

[3] A. L. Olsen, "An evolutionary algorithm to solve the joint replenishment problem using direct grouping," Computers and Industrial Engineering, vol. 48, no. 2, pp. 223-235, 2005.

[4] I. K. Moon and B. C. Cha, "The joint replenishment problem with resource restriction," European Journal of Operational Research, vol. 173, no. 1, pp. 190-198, 2006.

[5] M. Khouja and S. Goyal, "A review of the joint replenishment problem literature: 1989-2005," European Journal of Operational Research, vol. 186, no. 1, pp. 1-16, 2008.

[6] H. Qu, L. Wang, and Y. R. Zeng, "Modeling and optimization for the joint replenishment and delivery problem with heterogeneous items," Knowledge-Based Systems, vol. 54, pp. 207-215, 2013.

[7] L. Wang, H. Qu, Y. Li, and J. He, "Modeling and optimization of stochastic joint replenishment and delivery scheduling problem with uncertain costs," Discrete Dynamics in Nature and Society, vol. 2013, Article ID 657465, 12 pages, 2013.

[8] L. Wang, H. Qu, S. Liu, and C. X. Dun, "Modeling and optimization of the multiobjective stochastic joint replenishment 
and delivery problem under supply chain environment," The Scientific World Journal, vol. 2013, Article ID 916057, 11 pages, 2013.

[9] N. C. Büyükkaramikli, Ü. Gürler, and O. Alp, "Coordinated logistics: joint replenishment with capacitated transportation for a supply chain," Production and Operations Management, vol. 23, no. 1, pp. 110-126, 2013.

[10] L. G. Cui, L. Wang, and J. Deng, "RFID technology investment evaluation model for the stochastic joint replenishment and delivery problem," Expert Systems with Applications, vol. 41, no. 4, pp. 1792-1805, 2014.

[11] B. C. Cha and I. K. Moon, "The joint replenishment problem with quantity discounts under constant demand," OR Spectrum, vol. 27, no. 4, pp. 569-581, 2005.

[12] I. K. Moon, S. K. Goyal, and B. C. Cha, “The joint replenishment problem involving multiple suppliers offering quantity discounts," International Journal of Systems Science, vol. 39, no. 6, pp. 629-637, 2008.

[13] B. C. Cha and J. H. Park, “The joint replenishment and delivery scheduling involving multiplesuppliers offering different quantity discounts," in Proceedings of the International Conference on Computers and Industrial Engineering (CIE '09), pp. 52-56, Troyes, France, July 2009.

[14] S.-L. Hsu, "Optimal joint replenishment decisions for a central factory with multiple satellite factories," Expert Systems with Applications, vol. 36, no. 2, pp. 2494-2502, 2009.

[15] T.-H. Chen and J.-M. Chen, "Optimizing supply chain collaboration based on joint replenishment and channel coordination," Transportation Research Part E: Logistics and Transportation Review, vol. 41, no. 4, pp. 261-285, 2005.

[16] L. Wang, J. He, and Y.-R. Zeng, "A differential evolution algorithm for joint replenishment problem using direct grouping and its application," Expert Systems, vol. 29, no. 5, pp. 429-441, 2012.

[17] L. Wang, C.-X. Dun, W.-J. Bi, and Y.-R. Zeng, "An effective and efficient differential evolution algorithm for the integrated stochastic joint replenishment and delivery model," KnowledgeBased Systems, vol. 36, pp. 104-114, 2012.

[18] J. Geunes and P. M. Pardalos, Supply Chain Optimization, Kluwer Academic Publishers, 2004.

[19] J. Geunes, P. M. Pardalos, and E. Romeijn, Supply Chain Management: Models, Applications, and Research Directions, Kluwer Academic Publishers, 2002.

[20] P. M. Pardalos, A. Migdalas, and G. Baourakis, Supply Chain and Finance, World Scientific, 2004.

[21] L. Wang, Q.-L. Fu, and Y.-R. Zeng, "Continuous review inventory models with a mixture of backorders and lost sales under fuzzy demand and different decision situations," Expert Systems with Applications, vol. 39, no. 4, pp. 4181-4189, 2012.

[22] Y. H. Li, H. Guo, L. Wang, and J. Fu, "A hybrid genetic-simulated annealing algorithm for the location-inventory-routing problem considering returns under e-supply chain environment," The Scientific World Journal, vol. 2013, Article ID 125893, 10 pages, 2013.

[23] L. Wang, R. Yang, P. M. Pardalos, L. Qian, and M. Fei, "An adaptive fuzzy controller based on harmony search and its application to power plant control," International Journal of Electrical Power and Energy Systems, vol. 53, no. 1, pp. 272-278, 2013.

[24] L. Cui, L. Wang, J. Deng, and J. Zhang, "A new improved quantum evolution algorithm with local search procedure for capacitated vehicle routing problem," Mathematical Problems in Engineering, vol. 2013, Article ID 159495, 17 pages, 2013.

[25] J. Pei, X. B. Liu, P. M. Pardalos, W. J. Fan, S. Yang, and L. Wang, "Application of an effective modified gravitational search algorithm for the coordinated scheduling problem in a two-stage supply chain," International Journal of Advanced Manufacturing Technology, vol. 70, no. 1-4, pp. 335-348, 2014.

[26] F. S. Al-Anzi and A. Allahverdi, "A self-adaptive differential evolution heuristic for two-stage assembly scheduling problem to minimize maximum lateness with setup times," European Journal of Operational Research, vol. 182, no. 1, pp. 80-94, 2007.

[27] L. Wang, H. Qu, T. Chen, and F. P. Yan, "An effective hybrid self-adapting differential evolution algorithm for the joint replenishment and location-inventory problem in a three-level supply chain," The Scientific World Journal, vol. 2013, Article ID 270249, 11 pages, 2013.

[28] R. Storn and K. Price, "Differential evolution-a simple and efficient heuristic for global optimization over continuous spaces," Journal of Global Optimization, vol. 11, no. 4, pp. 341359, 1997.

[29] Q.-K. Pan, L. Wang, and B. Qian, "A novel differential evolution algorithm for bi-criteria no-wait flow shop scheduling problems," Computers \& Operations Research, vol. 36, no. 8, pp. 2498-2511, 2009.

[30] J.-M. Chen and T.-H. Chen, "The multi-item replenishment problem in a two-echelon supply chain: the effect of centralization versus decentralization," Computers and Operations Research, vol. 32, no. 12, pp. 3191-3207, 2005.

[31] A. Salman, A. P. Engelbrecht, and M. G. H. Omran, "Empirical analysis of self-adaptive differential evolution," European Journal of Operational Research, vol. 183, no. 2, pp. 785-804, 2007.

[32] Y. Wang, B. Li, and T. Weise, "Estimation of distribution and differential evolution cooperation for large scale economic load dispatch optimization of power systems," Information Sciences, vol. 180, no. 12, pp. 2405-2420, 2010.

[33] J. Brest, S. Greiner, B. Bošković, M. Mernik, and V. Zumer, "Self-adapting control parameters in differential evolution: a comparative study on numerical benchmark problems," IEEE Transactions on Evolutionary Computation, vol. 10, no. 6, pp. 646-657, 2006.

[34] F. Neri and V. Tirronen, "Recent advances in differential evolution: a survey and experimental analysis," Artificial Intelligence Review, vol. 33, no. 1-2, pp. 61-106, 2010.

[35] L. Wang, C.-X. Dun, C.-G. Lee, Q.-L. Fu, and Y.-R. Zeng, "Model and algorithm for fuzzy joint replenishment and delivery scheduling without explicit membership function," International Journal of Advanced Manufacturing Technology, vol. 66, no. 9-12, pp. 1907-1920, 2013.

[36] Y.-R. Zeng, L. Wang, and J. He, "A novel approach for evaluating control criticality of spare parts using fuzzy comprehensive evaluation and GRA," International Journal of Fuzzy Systems, vol. 14, no. 3, pp. 392-401, 2012. 


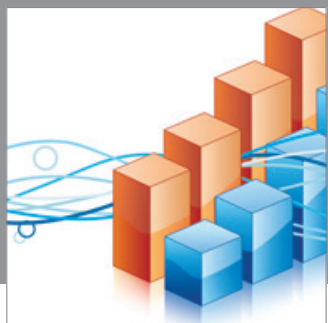

Advances in

Operations Research

mansans

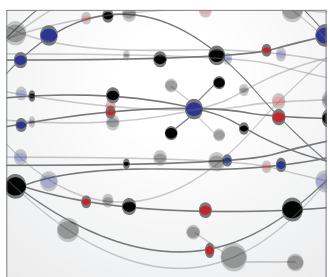

The Scientific World Journal
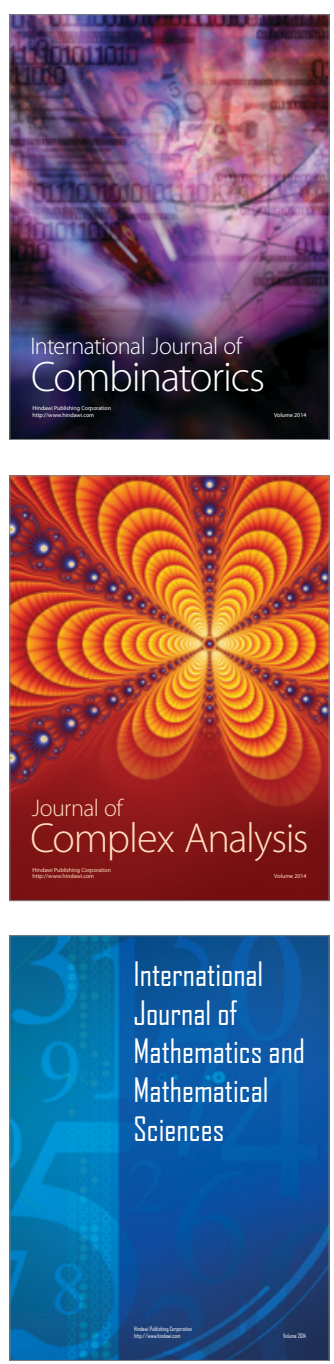
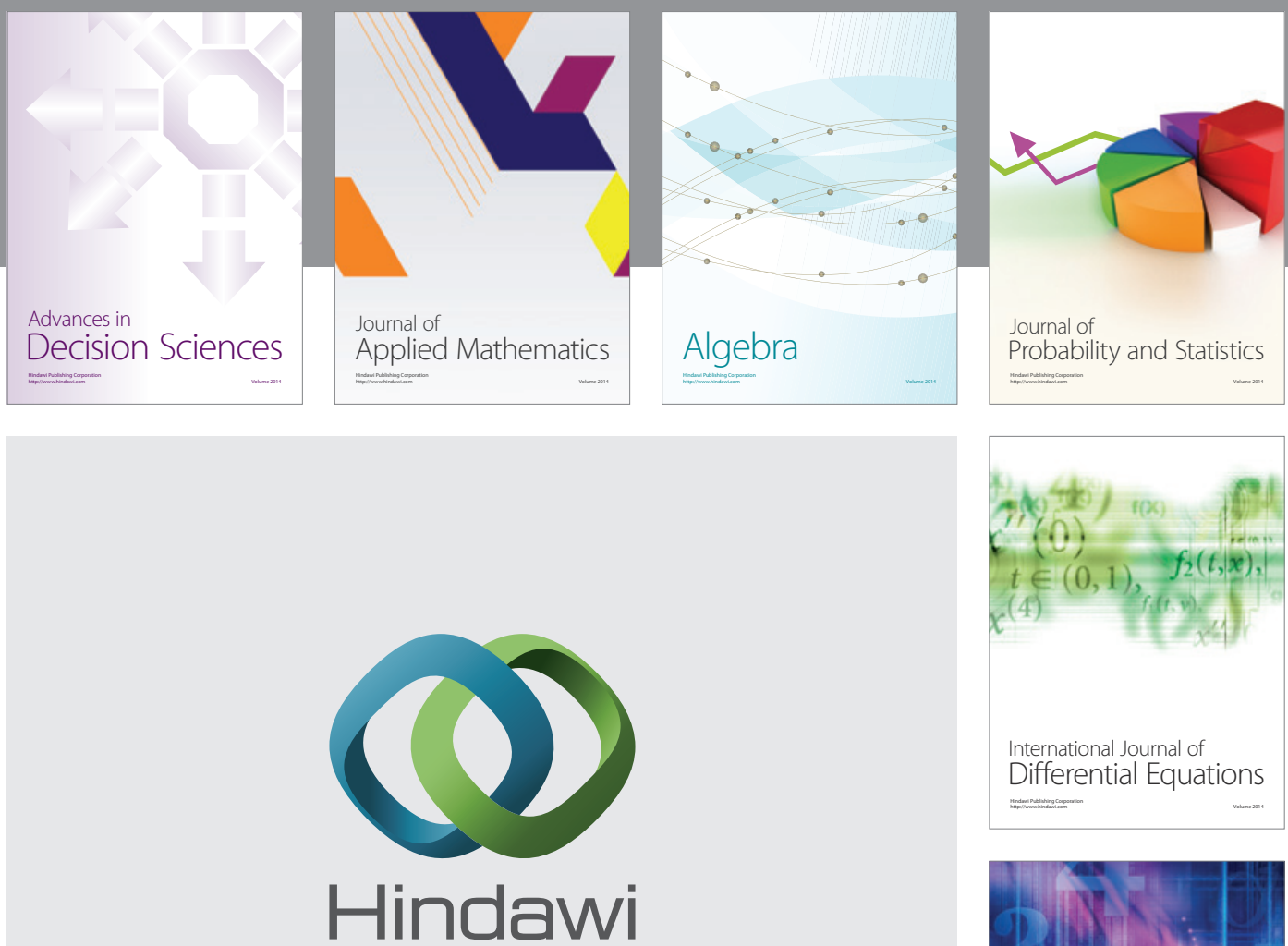

Submit your manuscripts at http://www.hindawi.com
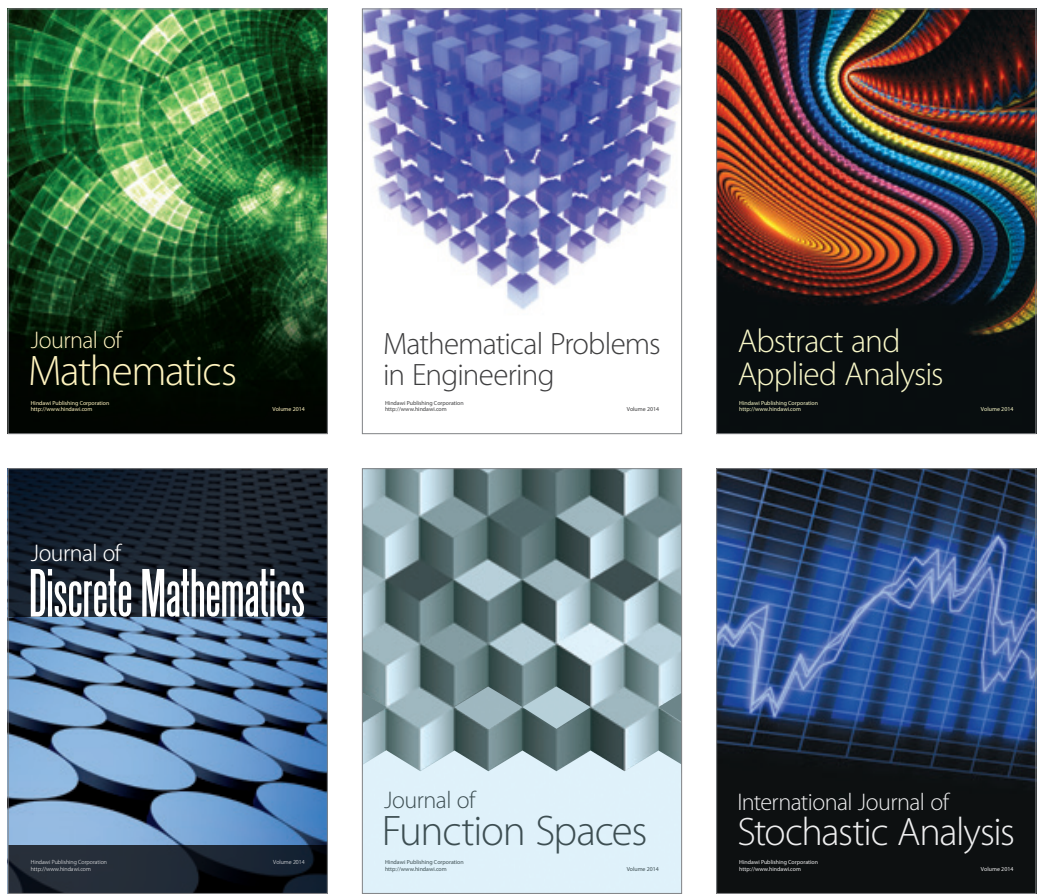

Journal of

Function Spaces

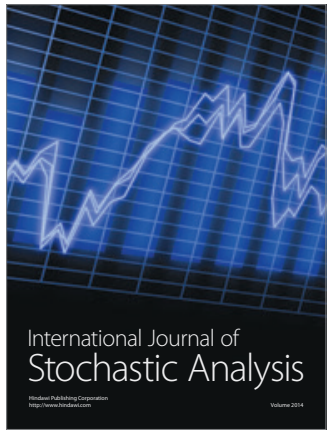

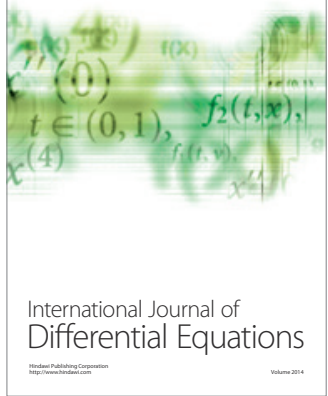
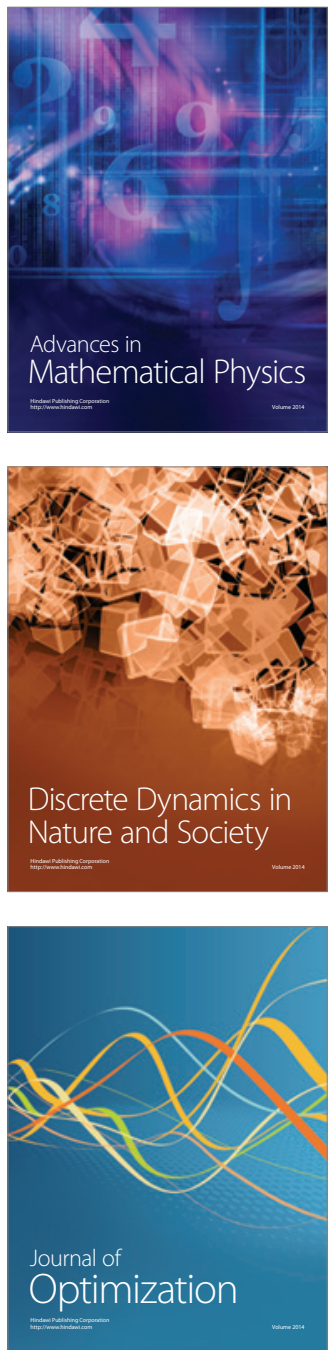